\title{
Next-generation sequencing panel for diagnosis and management of chronic neutrophilic leukaemia: a case report
}

KY Mak', MB, ChB, CH Au², PhD, TL Chan², PhD, Edmond SK Ma², MD, FHKAM (Pathology), Eudora YD Chow', MB, BS, FHKAM (Pathology), SY Lin ${ }^{3}$, MB, BS, FHKAM (Medicine), William WL Choi ${ }^{1 *}$, MB, BS, FHKAM (Pathology)

${ }^{1}$ Department of Pathology, United Christian Hospital, Kwun Tong, Hong Kong

${ }^{2}$ Department of Pathology, Hong Kong Sanatorium \& Hospital, Happy Valley, Hong Kong

${ }^{3}$ Department of Medicine and Geriatrics, United Christian Hospital, Kwun Tong, Hong Kong

*Corresponding author: wlchoi@hotmail.com

Hong Kong Med J 2019;25:248-50

https://doi.org/10.12809/hkmj176959

\section{Case report}

An 80-year-old woman presented to our hospital with mild headache in November 2015. She had a history of hypertension, diabetes mellitus, and hyperlipidaemia. She was febrile $\left(38.6^{\circ} \mathrm{C}\right)$ but did not appear septic. Abdominal examination revealed mild splenomegaly but no hepatomegaly and there were no focal neurological signs or suggestions of other organ involvement. A full blood count showed leukocytes $124.0 \times 10^{9} / \mathrm{L}$ (neutrophils $120.3 \times 10^{9} / \mathrm{L}$, lymphocytes $2.5 \times 10^{9} / \mathrm{L}$, monocytes $1.2 \times 10^{9} / \mathrm{L}$ ); haemoglobin $7.8 \mathrm{~g} / \mathrm{dL}$, mean corpuscular volume $89.1 \mathrm{fL}$; and platelets $384 \times 10^{9} / \mathrm{L}$. The blood film showed marked neutrophilia, occasional myelocytes, and absolute basophilia but no blasts. The neutrophils showed toxic granules and were not dysplastic (Fig 1). Plain radiographs of the chest, kidney, ureter, and urinary bladder did not reveal any abnormalities. Bacterial cultures of the blood and urine did not reveal any septic foci. Because of the marked neutrophilia, the patient was initially treated for bacterial sepsis with empirical intravenous amoxicillin with clavulanic acid. Subsequent ultrasonography of the abdomen

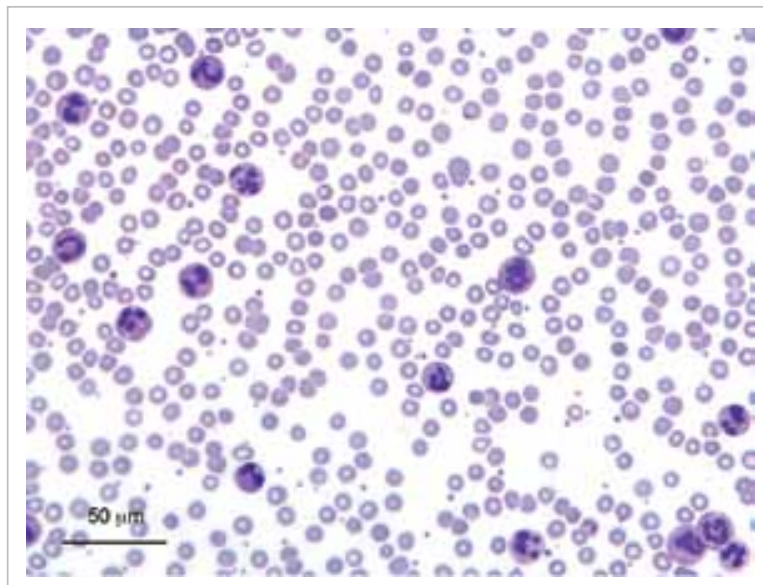

FIG I. Peripheral blood film showed marked neutrophilia, and the neutrophils showed toxic granules but were not dysplastic (May-Grünwald-Giemsa, ×400) confirmed the splenomegaly $(15.1 \mathrm{~cm})$ but no other space-occupying lesions. The low-grade fever soon subsided after admission and she remained afebrile and non-septic, although the marked neutrophilia persisted.

A bone marrow biopsy revealed marked hypercellularity, primarily due to markedly increased granulopoiesis with left-shift in maturation but no increase in blasts. Erythropoiesis was active and normoblastic. Megakaryocytes were moderately increased with some being large and hyperlobulated. No overt dysplasia was seen. Cytogenetic karyotyping showed a normal karyotype. Reverse transcription polymerase chain reaction (PCR) for $B C R-A B L 1$ and allele-specific PCR for Janus kinase 2 (JAK2) V617F mutation analysis were negative. In view of the clinical and laboratory picture of a possible myeloproliferative neoplasm, and the lack of a clonal marker detected by the molecular tests, we sought to utilise a next-generation sequencing (NGS) panel (TruSight Myeloid Sequencing Panel; Illumina, San Diego [CA], United States) to look for possible mutations in selected exons of 54 different genes commonly implicated in myeloid neoplasms, in accordance with a previously published protocol. ${ }^{1}$ Deep sequencing by this panel returned three pathogenic mutations: colony-stimulating factor 3 receptor (CSF3R) c.1853C $>$ T; p.Thr618Ile or T618I at variant allele frequency (VAF) of $43.9 \%$ (Fig 2a), serine/arginine-rich splicing factor 2 (SRSF2) c.284C >T; p.Pro95Leu (NM_003016.4) or P95L at VAF of $49.6 \%$, and additional sex combs like 1 (ASXL1) truncating mutation c.1934dupG; p.Gly646Trpfs*12 (NM_015338.5) at VAF of 34.1\%. The CSF3R T618I mutation was further confirmed by Sanger sequencing (Fig $2 \mathrm{~b}$ ), thus prompting the diagnosis of chronic neutrophilic leukaemia (CNL). Although the CSF3R mutation rendered the disease amenable to ruxolitinib, the patient deteriorated rapidly and died of sudden severe gastrointestinal bleeding 12 days after admission and before specific therapy could be contemplated. 


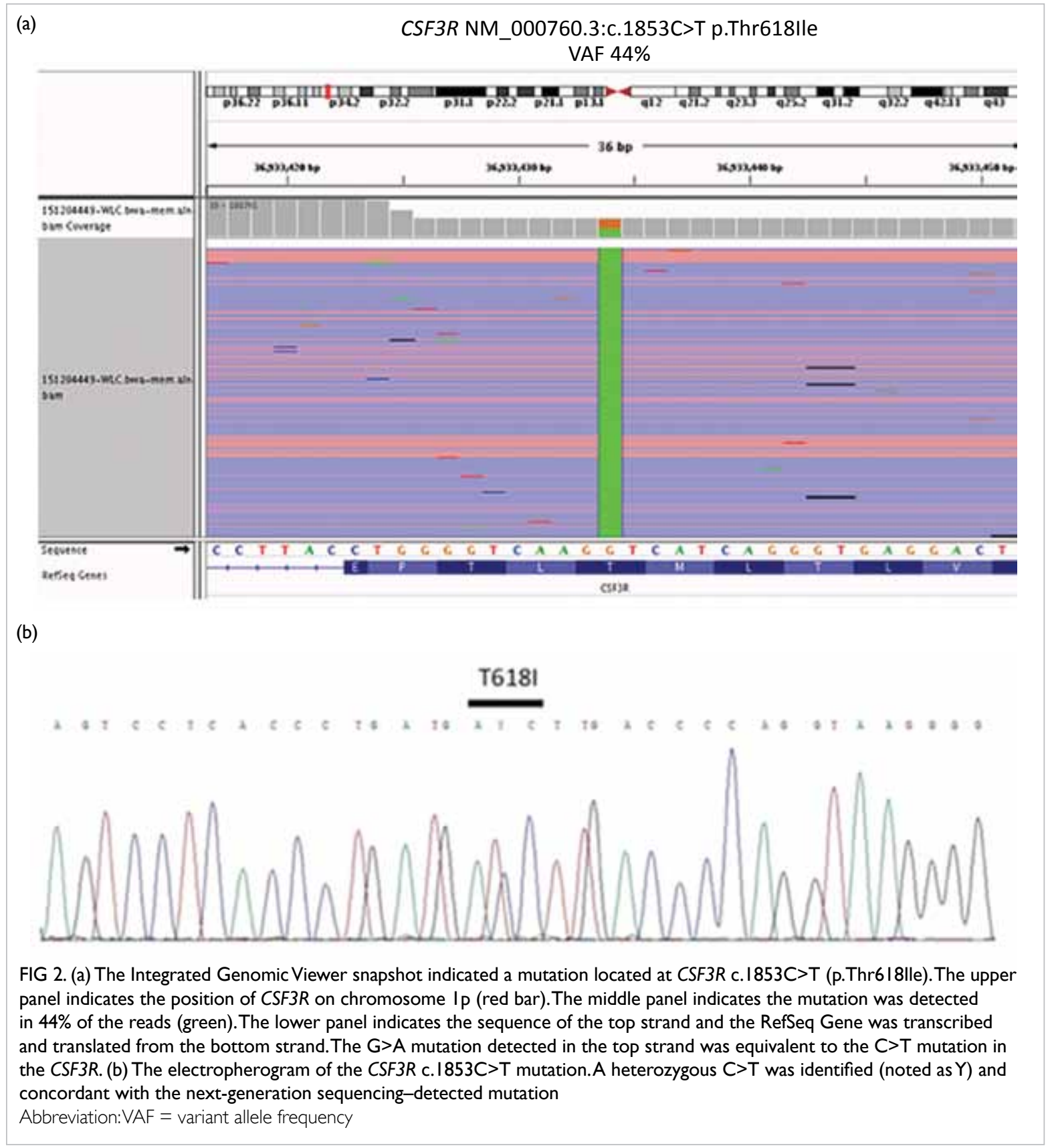

\section{Discussion}

Several clinical features in this patient hinted at differential diagnoses other than bacterial infection or acute inflammation. Apart from fever, there were no other clinical features related to the presenting symptom of headache. Splenomegaly, a common feature of myeloproliferative neoplasms, was present. The absolute neutrophil count was very high, yet no clinical features of sepsis were found on physical examination or from investigations.

Since 2013, understanding of the molecular genetics of CNL has been dramatically changed by the discovery of CSF3R mutations in around 80\% of cases. ${ }^{2}$ The CSF3R encodes a transmembrane receptor for granulocyte colony-stimulating factor 3 , and plays a crucial role in the differentiation and maturation of neutrophils. ${ }^{2}$ The $\mathrm{CNL}$-associated mutations in CSF3R activate the receptor and promote the proliferation and differentiation of neutrophils, leading to the marked neutrophilia that characterises the CNL disease phenotype. ${ }^{3}$ There are two major types of CSF3R mutations in CNL. The first encompasses point mutations in the extracellular or transmembrane domains, of which the T618I mutation is the most common and comprises the majority of mutations in CNL. The second type of CSF3R mutation comprises nonsense or frameshift mutations leading to a premature stop 
codon and truncation of the cytoplasmic domain of the receptor. ${ }^{2}$

Mutations of other genes have also been reported in CNL. These can be grouped as SET binding protein 1 (SETBP1) mutations, spliceosome mutations (eg, SRSF2), epigenetic modifier mutations (eg, ASXL1), and signalling mutations (eg, JAK2). ${ }^{3}$ One previous study found that SRSF2 mutations occurred in three of 14 cases of CNL (21\%). The SRSF2 mutations were previously associated with a worse prognosis in chronic myelomonocytic leukaemia, but its effects on CNL are unclear. ${ }^{3} \mathrm{~A}$ significant proportion of CNL patients have been shown to harbour ASXL1 (30\%-60\%). Similar to mutations in other myeloid malignancies, ASXL1 mutations in CNL have been shown to confer a poor prognosis. $^{3}$

Although these other mutations have not been incorporated into the World Health Organization 2016 diagnostic criteria for CNL, these data suggest that some may show prognostic value. Additionally, different CSF3R mutations may allow different therapeutic approaches (see below). Because Sanger sequencing of an increasing number of genes leads to substantial increases in the required time, resources, and necessary amount of DNA, we sought to explore an NGS panel to interrogate these genes simultaneously more efficiently and cost-effectively. Of note, the panel includes genes that are important for the diagnosis of myeloproliferative neoplasm $(J A K 2$, calreticulin [CALR] and myeloproliferative leukaemia protein $[M P L])$, plus genes that are frequently reported in CNL (CSF3R, SETBP1, $S R S F 2, A S X L 1)$. Therefore, compared with Sanger sequencing, NGS panels are a more efficient and powerful means to enable comprehensive genomic profiling of individual CNL cases, utilising a smaller amount of DNA.

With recent discoveries in the molecular pathogenesis of CNL, new therapeutic approaches that target the CSF3R signalling pathway-related SRC family and JAK-kinase pathways have emerged. The SRC signalling pathway is activated by truncation mutations of CSF3R leading to sensitivity to dasatinib, while the JAK-STAT pathway is activated by membrane proximal mutations of CSF3R leading to sensitivity to ruxolitinib. ${ }^{2}$ Although there are few reported cases of these agents, ${ }^{2,4}$ they represent significant breakthroughs in the management of CNL. This case of a rare myeloproliferative neoplasm demonstrates how advances in understanding of the molecular pathogenesis of a disease open up new routes for the development of effective novel therapeutic strategies.

\section{Author contributions}

All authors had full access to the data, contributed to the study, approved the final version for publication, and take responsibility for its accuracy and integrity.

Concept and design of study: WWL Choi.

Acquisition of data: KY Mak, CH Au, TL Chan, ESK Ma, SY Lin, WWL Choi.

Analysis or interpretation of data: $\mathrm{KY} \mathrm{Mak,} \mathrm{CH} \mathrm{Au}$, TL Chan, ESK Ma, WWL Choi.

Drafting of the manuscript: KY Mak, WWL Choi.

Critical revision for important intellectual content: $\mathrm{CH} \mathrm{Au}$, TL Chan, ESK Ma, EYD Chow, SY Lin.

\section{Conflicts of interest}

All authors have no conflicts of interest to declare.

\section{Funding/support}

This research received no specific grant from any funding agency in the public, commercial, or not-for-profit sectors.

\section{References}

1. Au CH, Wa A, Ho DN, Chan TL, Ma ES. Clinical evaluation of panel testing by next-generation sequencing (NGS) for gene mutations in myeloid neoplasms. Diagn Pathol 2016;11:11.

2. Maxson JE, Gotlib J, Pollyea DA, et al. Oncogenic CSF3R mutations in chronic neutrophilic leukemia and atypical CML. N Engl J Med 2013;368:1781-90.

3. Maxson JE, Tyner JW. Genomics of chronic neutrophilic leukemia. Blood 2017;129:715-22.

4. Stahl M, Xu ML, Steensma DP, Rampal R, Much M, Zeidan AM. Clinical response to ruxolitinib in CSF3R T618mutated chronic neutrophilic leukemia. Ann Hematol 2016;95:1197-200. 